\title{
ORIE Research Summar'y

\section{How to strengthen an Infant and Young Child Feeding programme in northern Nigeria}

This summary highlights findings and recommendations from operations research into how to strengthen the implementation of an Infant and Young Child Feeding (IYCF) programme and to motivate behaviour change to improve IYCF practices in northern Nigeria.

\section{Introduction}

The prevention of undernutrition via IYCF is one of four outputs of the UK Department for International Development funded Working to Improve Nutrition in Northern Nigeria (WINNN) programme. This output aims to prevent undernutrition by promoting evidencebased IYCF recommendations, including:

- exclusive breastfeeding until an infant is six months of age;

- feeding a diverse diet of nutritious foods and breast milk from 6 to 23 months of age;

- feeding a sick child;

- and good hand washing practices.

WINNN is implemented by UNICEF, Action Against Hunger (ACF) and Save the Children International (SCI), in partnership with the Federal Government of Nigeria and the State Governments of Jigawa, Katsina, Kebbi, Yobe and Zamfara. $\mathrm{SCl}$ is responsible for its implementation in Zamfara, Kebbi and Katsina states, and ACF is responsible for its implementation in Jigawa and Yobe states. Each of the five WINNN states implements the IYCF programme in three Local Government Areas (LGAs).

\section{Research questions and objectives}

The key operational research questions identified with WINNN for the IYCF programme were:

a. How to strengthen programme implementation and its integration into the primary health care system?

b. How to motivate behaviour change to improve infant and young child feeding practices?

The objectives of the research were:

1. To determine the opinions of health sector officials and IYCF programme beneficiaries about the implementation, benefits and challenges of the programme.

2. To understand the experiences, motivation and long term commitment of IYCF programme health workers and community volunteers.

3. To determine how to strengthen IYCF programme implementation to facilitate its integration into routine health services and meet the needs of IYCF programme functionaries and beneficiaries
«The IYCF programme was viewed positively because of the perceived visibility of the health benefits, including less episodes of diarrhoea, and consequent savings on health care.»)

\section{The WINNN IYCF programme includes:}

1. Facility-based IYCF counselling by health workers (HWs) for pregnant women and mothers with an infant less than two years of age;

2. Community-based IYCF individual and support group counselling by community volunteers (CVs) for pregnant women and mothers with an infant less than two years of age;

3. Community-based meetings with fathers of infants less than two years of age and grandmothers, facilitated by CVs. 


\section{ORIE Research Summary}

4. To determine the barriers, motivators and facilitating factors for the adoption of the improved infant feeding practices being promoted in the IYCF programme.

5. To determine how to support IYCF beneficiaries and motivate behaviour change towards improved infant feeding practices.

\section{Research approach}

The IYCF operations research was conducted in September 2014 in two LGAs in two of the five WINNN focal states in northern Nigeria: Katsina and Kebbi. Data were collected through 44 focus group discussions and 27 in-depth interviews.

The focus group discussions in urban and rural communities were facilitated with women who had and had not participated in the community-based IYCF counselling / support group sessions; grandmothers, traditional birth attendants and husbands of women who were eligible for the IYCF programme; and IYCF programme community volunteers (male and female).

In-depth interviews were facilitated with state level officials, including the Directors of Primary Health Care, Nutrition Officers, Health Education Officers and Reproductive Health Officers; health workers participating in the IYCF programme; and state level WINNN IYCF Advisors.

The results presented are based upon the perceptions and experiences as reported by those interviewed and the interpretation of these by the research team.

\section{Findings}

The IYCF programme was viewed positively because of the perceived visibility of the health benefits, including less episodes of diarrhoea, and consequent savings on health care. These perceived health benefits were the main reason fathers and state level officials were supportive of programme continuation and expansion.

\section{IYCF programme implementation}

At the community level, the IYCF programme is designed to be delivered by CVs through support groups or individual level counselling.
Preferences for the CV facilitated support group or individual counselling sessions were varied amongst interviewees. Support group counselling was the preference of grandmothers, CVs and some mothers interviewed because it increased confidence, reinforced attitudes, understanding and spread of the messages through peer-to- peer interactions. Individual counselling was the preference of other mothers and health workers interviewed because it addressed individual concerns. These perceptions concur with opinions in other countries.

Even though the data suggest $\mathrm{CV}$ s are performing IYCF sensitisation in the communities, it was not clear whether the support groups were held as planned i.e., it was unclear as to whether the same women were meeting regularly.

There was broad consensus among health sector officials interviewed that the IYCF programme was well integrated into existing health services, in particular with antenatal care (ANC) and immunisation services. Integration was mainly perceived as IYCF health messages being delivered and/or reinforced during other health services, and key messages about other health programmes being delivered through IYCF. There was a perception from some health sector officials that IYCF had improved the quality of the health messages being delivered in ANC. There also appeared to be an active complementary referral system occurring between the hospital and community components of the IYCF programme.

One important finding from this study, which is based on the perceptions of some health sector officials, is that the IYCF community-based programme has resulted in higher attendance at ANC and childhood immunisations; coverage of which are extremely low in northern Nigeria. In terms of the IYCF programme reaching as high a proportion of pregnant women, mothers and young children as possible, the community component is clearly very important as access to routine preventative programmes in health facilities is low. Furthermore, if IYCF truly does have the potential to increase coverage of ANC and/or childhood immunisations in northern Nigerian, then it is of major importance to the public health of the population.
«The IYCF programme was well integrated into existing health services, in particular with antenatal care and immunisation services. 》) 


\section{ORIE Research Summary}

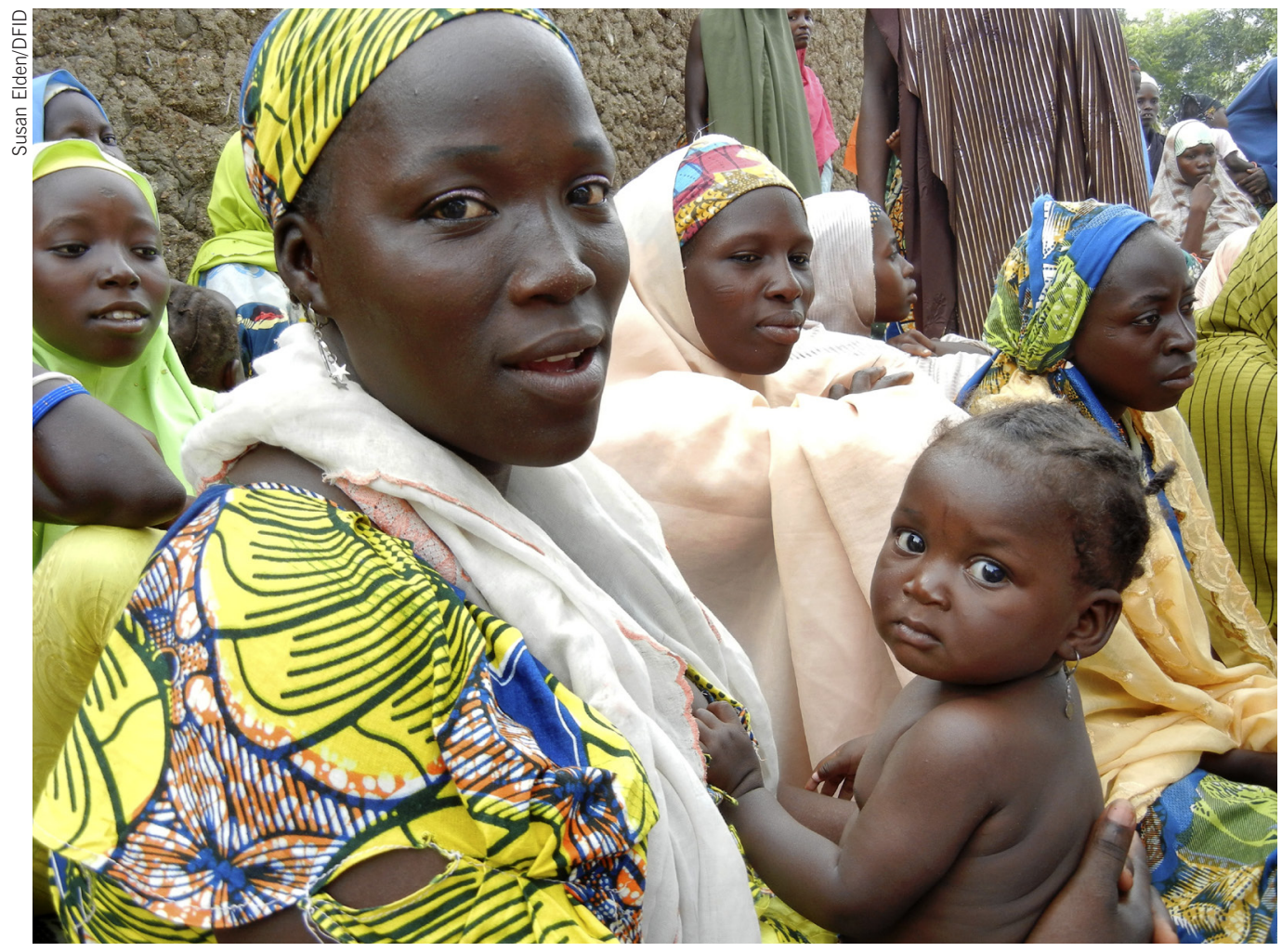

«Targeting influential household decision makers will also be important for overcoming key barriers to complementary feeding. 》》

A woman attends a health education session in northern Nigeria

Concerns were raised by fathers and health sector officials about the limited financial allowances given to the IYCF CVs, which was perceived as a threat to long-term programme sustainability. The constraints of time, the need for a transport allowance and remuneration identified by the $\mathrm{CV}$ s are not unique to the Nigerian IYCF programme. Further research is needed to evaluate appropriate mixes of material or non-material incentives for $\mathrm{CVs}$, to strengthen their motivation and commitment to the WINNN supported IYCF programme, as high CV attrition is disruptive and costly.

Recommendations from CVs included strengthening the refresher training and supportive supervision components of the programme. Successful scale-up of IYCF elsewhere has been partially attributed to strong refresher training and supportive supervision systems. Supportive supervision, in particular, was perceived by CVs as important for strengthening their motivation, confidence and skills, in delivering the programme; and for increasing the perceived importance of the programme and the community's confidence in it.

Political commitment, partnerships, IYCF champions and advocacy, are also key components for successful scale-up, as reported elsewhere and in the ORIE qualitative evaluation report.

\section{Behaviour change strategy and messages}

Information materials and small scale media behaviour change strategies are being used in the IYCF programme, as evidenced by beneficiary knowledge of the recommended IYCF practices, and positive comments about counselling cards (materials) and pictures of healthy children (small scale media). It was less clear, however, whether counselling behaviour change techniques are being used, because few strategies to overcome barriers to behaviour change were reported by programme beneficiaries, and interactions with mothers were usually described as giving advice rather than as listening to mothers' concerns, helping them to decide what is best to do and strengthening their confidence to do it.

The concept of counselling is relatively new in Nigeria, so further training and supportive supervision on the use of counselling techniques, especially in the initial phases of the IYCF programme, are likely needed to reinforce their use. 


\section{ORIE Research Summary}

For exclusive breastfeeding, an important underlying barrier is a cultural belief that infants need water to survive. This strongly held belief means there is some distrust of the programme, which respondents felt could be overcome by repeated messaging and behaviour change strategies that target social norms, especially among authoritative family decision makers, such as fathers and grandmothers. Targeting influential household decision makers will also be important for overcoming key barriers to complementary feeding, i.e. the availability and affordability of recommended foods, especially animal source foods, because husbands 'often control the family's food budget'.

Strong and clear views were expressed on effective messaging for both acceptance of the IYCF content and encouraging behaviour change:

- keep IYCF messaging distinct and separate from family planning;

- reassure mothers and family members that a large proportion of breast milk is water and therefore exclusive breastfeeding does not mean that a child gets no water;

- focus on a limited number of locally available, affordable foods for complementary feeding;

- and emphasise visible changes to a mother's or child's health after the adoption of recommended practices.

\section{Recommendations for WINNN}

1. Review monitoring records to determine and strengthen the implementation of the community component of IYCF, as required.

2. Develop and test material and/or non-material incentives for CVs, such as providing of transportation allowance for them to reach remote areas or facilitating indirect mechanisms that would serve to improve livelihoods, the status of an IYCF CV or reduce their opportunity costs.

3. Review strategies for CV training and strengthen supportive supervision, especially in relation to counselling techniques and facilitation of support groups.

4. Translate counselling cards into local languages using pictures of readily available local foods.

5. Prioritise the promotion of a small number of context-specific doable actions rather than a broad range of improved practices.

6. Reinforce and strengthen the use of examples of real healthy children who have been exclusively breastfed to enhance the acceptability of the messages and adoption of the behaviour change.

7. Adopt multiple behaviour change techniques, including food demonstrations and professionally developed mass media messages tailored for men.

\section{ORIE and WINNN}

ORIE is an independent component of the UK Government's Department for International Development (DFID) funded Working to Improve Nutrition in Northern Nigeria (WINNN) programme. WINNN is working to improve the nutritional status of 6.2 million children under five years of age in five states of northern Nigeria. ORIE is carrying out research to determine the impact of WINNN and generate important research on key evidence gaps regarding solutions to undernutrition in northern Nigeria.

\section{Credits}

This ORIE Research Summary is based on the IYCF operations research report by Olutayo Adeyemi, Wasiu A. Afolabi, Elaine Ferguson, Jenna Hoyt, Hussiani Lawal, Rasheed A. Okunola, Jayne Webster, and Cynthia Yohanna-Dzingina. Readers are encouraged to quote and reproduce material from ORIE Research Summaries in their own publication. In return, ORIE requests due acknowledgement and quotes to be referenced as above.

ORIE cannot be held responsible for errors or any consequences arising from the use of information contained in this publication. Any views and opinions expressed do not necessarily reflect those of DFID.

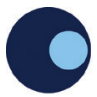

Oxford Policy Management

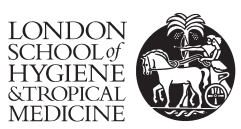

UNIVERSTISY OF IBADAN
Save the Children UKaid

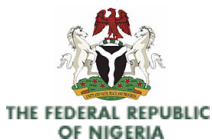

ORIE is run by a consortium of UK and Nigerian organisations. In the UK: Oxford Policy Management, Institute of Development Studies, London School of Hygiene and Tropical Medicine, Save the Children UK; in Nigeria: Food Basket Foundation International, University of Ibadan. ORIE works in partnership with the Federal Government of Nigeria.

For any further information about ORIE please email orienigeria@orie-ng.org or see the website heart-resources.org/tag/orie 